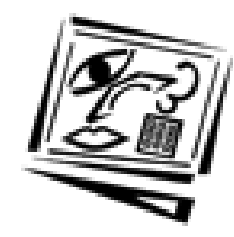

\title{
Interactivity on stage: Strategies for learner-designer communication
}

\author{
Rod Sims \\ Southern Cross University
}

\section{Introduction}

The word interactive, when used to described computer based learning resources, has tended to imply better experiences, more active learning, enhanced interest and motivation. But despite the investment in productions to date, this interactive condition has not been consistently realised. Although the surge in internet based communications and collaborative learning activities has extended the opportunities for human-human communication, the complexity of learner-computer interactivity has yet to be fully unravelled.

This paper examines the relationship between the independent learner and computer based learning resources, which continue to be integral to educational delivery, especially in the training sector. To place interactivity in context, the first part of the discussion focuses on the major dimensions of interactivity and the different ways they have been characterised in computer based learning environments. These dimensions demonstrate the many ways that interactivity can be interpreted and the critical role that design and development plays in creating effective interactive encounters. The second part of the paper reviews the way storytelling structures and narrative have been promoted as effective strategies for enhancing comprehension and engagement in computer based learning applications. The way in which the interactivity and narrative are linked becomes critical to achieving this outcome.

Extending the use of a narrative within interactive media to include elements of performance and theatre, the third part of the discussion proposes that by conceptualising the learner as actor, a form of learnerdesigner communication can be established. Integrating this approach with elements of conversational and communication theory provides a 
context in which the learner-computer interface is transcended by that of learner and designer. Enabling this form of communication with the independent learner is suggested as a means to enhance computer based learning environments.

\section{Dimensions of interactivity}

\section{Interactivity - hero or villain?}

Interactivity refers to the facilities provided by a computer based application to provide the user with both control of the process and communication with content. This communication involves both the user initiating an action and the computer responding to that action. The computer's role with interactivity is to enable the processing power to interpret and respond to a user's action, thereby generating a form of human-computer dialogue. While interactivity as a concept has retained high profile throughout the history of computer based learning, its interpretation has undergone a metamorphosis and continues to be regarded as complex and difficult to define. For example, while acknowledging that computers can support an interactive experience, Heath (1995) concludes that interactivity is essentially a function of human-human communication and that its current use has resulted in "at best confusion and at worst deception". The argument presented in this paper is that if interactivity has generated confusion, then it is the responsibility of the designer to correct that anomaly, especially where the learner is working independently.

In contrast interactivity is portrayed as the distinguishing factor of the new media, with the assumption that "interactivity in a computer product means that the user, not the designer, controls the sequence, the pace, and most importantly, what to look at and what to ignore" (Kristof \& Satran, 1995:35). This implies to some extent that the value of the material is based on the user's motivation and objectives. However, Holmes (1995:12 ) identifies that the structure or design of the material is equally critical and that the interactivity embodied within a computer is dependent on that design:

Interactivity is the ability of a new media program, web site, kiosk or multimedia presentation, to allow its user to control the content in some manner ... Interactivity must involve, engage, and motivate the user to explore the product ... Bad interactivity happens. The user can be frustrated by muddled organization, too much information or poor instructions ... Interactive properties of new media should provide 
opportunities for the user: exploration, discovery, and collaboration. Well conceived interactivity knows its audience, understands their knowledge base, and uses terms and phrases that are commonly understood by the audience. Good interactivity also takes into account the situation of the audience.

Examining this perception reveals a number of major factors - first the user must be able to control the application and the application must be designed to engage the user; second the user must have some freedom and third the application must be designed for a specific audience. Interactivity, at least from this perspective, is partly about what the user does and part about how the application is designed. However, this perspective is at odds with the notion of the individual learner - are designers able to create applications that will match each member of a target group?

In proposing a nascent theory of interactivity Jaspers (1991) identified different levels ranging from the linear (information delivery) to the communicative (student initiated). However, the success of such a theory was dependent on the relationship between designer and developer and the perceived role of the learner. Emphasising the benefits of a learner centred environment the problematic and contradictory nature of interactivity was noted:

In fact, the expression of "interactive delivery is contradicto in terminis from the viewpoint of interaction and emancipation. Delivery implies a unidirectional relationship. In full interaction there can be no one sided relation; the student is not just at the end of a chain but also at its beginning. (Jaspers (1991:21)

To what extent the learner can play a proactive role while working with computer based learning resources becomes critical to the success of such applications and is considered in the final part of this paper. A related issue concerns developing a common understanding of interactivity. Schwier \& Misanchuk (1993) refer to the levels defined in the literature as arbitrary and non-descriptive. Aldrich, Rogers \& Scaife (1998) refer to interactivity as ubiquitous and Plowman (1996b) considers the excesses of physical interactions as gratuitous. The confused state of interactivity was also acknowledged in the following:

Though lauded by many for its ability to handle user inputs, there is little consensus with regard to the design of human computer interactions. Indeed, disagreement even exists about the meaning of the terms interactive as applied to emerging technologies. Researchers have described fundamentally different perspectives on the roles of interactions, ranging from facilitating lesson navigation to supporting encoding of specific 
lesson content ... Research on interaction methods may be among the most critical ... The domain of possibilities has broadened substantially, yet little research has been advanced which might guide their design. (Hannafin, Hannafin, Hooper, Rieber \& Kini, 1996:385)

A critical approach to assessing interactivity was presented by Rose (1999) who, given the various taxonomies of interactivity coupled with an apparent lack of denotative value, set about a deconstruction of established understandings. Critical of the "good" versus "bad" mentality that pervades the field (active not passive, learner control not program control, constructivism not instructivism, hypermedia not linear delivery), Rose (1999:45) presented an interesting scenario that contradicts many perceptions of interactive environments:

Texts addressing the subject of interactivity ... privilege representations of highly motivated learners exploring the wonderful worlds of interactive instructional programs and making exciting discoveries. However, it is by virtue of a deconstructive reading that we can begin to see the shadowy figure of the disavowed other lurking behind these wide eyed adventurers: the shadow of a child sitting mesmerised and immobile before the computer, only her index finger on the mouse moving occasionally as a stream of images passes in a more or less predetermined sequence before her eyes.

Focusing explicitly on control Rose (1999) observed that although the words "learner control" have been interpreted as control by the learner, while grammatical comparisons (for example crowd control, weight control) tend to imply the opposite - control of the learner! The conclusion provides reinforcement for maintaining and extending research into making interactive learning work better:

That the field of educational computing is in need ... of internal critique is surely suggested by the fact that the very quality which is said to make computers unique and to justify their instructional use continues to defy definition. (Rose, 1999:48).

There remains a paradox of interactivity. On the one hand it is portrayed as an integral and critical component of computer based applications and on the other as ill defined, deceptive and difficult if not impossible to implement. Is interactivity the hero or villain of achieving success through computer based learning? To address this question the following dimensions of interactivity demonstrate its multi-faceted nature and the issues that are essential to consistently implementing effective interactions within computer based structures. 


\section{Interactivity as control}

One of the initial attempts to define interactivity was in terms of the control of videotape or videodisc. The extent to which the learner had control over the playing of video sequences was linked to the quality or level of the interactions (Iuppa (1984). Rhodes \& Azbell (1985) extended this in proposing three forms of interaction design strategies for computer assisted interactive video that integrated the control of content and structure - reactive (limited control of structure and content), coactive (a mix of limited and extended control of structure and content) and proactive (extended control of structure and content). As more control is provided to the learner the more they become proactive in determining the presentation forms of the content material, dependent nevertheless on the structures imposed by the designer. To extend this concept beyond interactive video, Rhodes \& Azbell (1985) proposed the transactive interaction, where users would communicate using a wide range of media to devise problem definitions, procedures and solutions. Other studies on the effects of different forms of interactivity on interactive video instruction concluded that "an improvement in learning may be expected in proportion to the amount and type of interactivity provided" (Schaffer \& Hannafin (1986:94) and that students had a preference for being able to control an interactive videodisc compared to a video presentation (Summers, 1990).

However, the value of the control provided to the learner is dependent of the consequences of the learner actions and the extent to which the application responds or adapts to the individual learner's actions.

\section{Interactivity as adaptation}

The extent to which the computer based learning application responds to a user's input is often referred to as adaptation. This adaptation can range from the program responding to a user's choice (such as a menu selection) to presenting material specifically structured according to the learner's prior responses or options. As described by Jonassen (1985:7), adaptation is part of the overall process of learner-computer interaction:

Interactive lessons are those in which the learner actively or overtly responds to information presented by the technology, which in turn adapts to the learner, a process more commonly referred to as feedback. The point is that interactive lessons require at least the appearance of two way communication. (Jonassen 1985:7) 
In this instance the adaptive capacity of the program is viewed in terms of a combination of the learner response and computer feedback, which in turn provides a form of communication. This process of questionresponse-feedback has been perceived by many writers as the essence of interactivity for computer based learning. For example, Steinberg (1991:100) observes that "question-response-feedback sequences help learners attain higher cognitive skills as well as factual information" and links the two elements of control and adaptation to the mechanics of interacting with the system (navigation) and the acquisition of knowledge and skills (learning). These descriptions identify two major components of interactivity - those which are program initiated and tell the learner what to do and those which are learner initiated, such as requests for help, information or explanation. This balance is also perceived as a means to achieve the goal of emulating human-human interaction in the computer medium, and thereby enhancing participation in the learning process.

\section{Interactivity as participation}

In comparison to the overt actions of controlling the pace and sequence of the learning or entering into a dialogue, the extent to which a learner is actually participating in and engaging with the content material focuses on the outcomes of the learning process. For example, Fenrich (1997:175) comments that:

Interactivity, or instructional features that promote active learning, provides critical support for increases in learning and retention in all educational activities ... Interaction implies active learner participation in the learning process .... an essential condition for effective learning ... failure to build interactivity into your program will reduce learning and retention.

This comment reflects a shift in emphasis from the overt nature of interactivity to the extent to which internal learning is facilitated. In providing a set of guidelines for interactivity, Fenrich (1997) suggests the following set of options:

- Thought provoking questions to enable the user to mentally process information;

- Active participation in a simulation or an educational game;

- Providing feedback, both detailed and elaborative;

- Building on current knowledge and experience, allowing learners to compare predictions and solutions;

- Learner control of pace and sequence; 
- Student comments and annotations, for later analysis and comparison;

- Learner modifications to the computer program, by including their own material.

In combination, the elements of control, adaptation and participation emphasise the potential for computer based technology to provide individualised learning experiences. Hannafin \& Peck (1988) include options such as identifying the learner by name, assigning a name to the computer, using relevant examples and integrating background information as strategies for making the experience meaningful to the learner. An additional element to consider is that of the experience level of the individual learner, and the extent to which the program caters for this experience (Weller, 1988). Extending interactivity beyond control, adaptation and participation led to the impact of interactive structures in terms of developing meaning - a shift from the physical actions of the learner to their internal.

\section{Interactivity for meaningful learning}

Hannafin (1989:167) observed that while rapidly developing technologies had "empowered instructional researchers and designers with unparalleled tools for manipulating instructional strategies" little of that potential had been exploited. In developing the concept of engagement, he suggested that "we are concerned with the manner in which instruction fosters cognitive engagement - the intentional and purposeful processing of lesson content" (Hannafin, 1989:170). To achieve this means focusing less on the physical, overt aspects of interaction and more on the cognitive, thinking activities of the embedded learning resource.

To provide a context for meaningful learning, Hannafin (1989) identified a set of five interactive functions (navigation, query, verification, elaboration, procedural control) and suggested a set of engaging activities to support these functions - fault free questions, queries, real time responding, notetaking, predicting/hypothesising, hypertext and cooperative dialogue. Although published over a decade ago the conclusions provide a useful guide for understanding interactivity:

It is no longer adequate to simply describe interactions in terms of either input technology employed or the physical characteristics of the responses made.... We need a richer understanding of the psychological

requirements associated with instructional tasks and responses, and a 
sense for how to extend design science beyond the methods that have evolved through the years. Hannafin's (1989:178)

Interactivity and meaningful learning has also been considered in terms of the schema model of human memory, where information is perceived as being stored in a web of interconnected nodes. "The strength of knowledge relies not simply on the number of nodes that exist, but more on the quality and number of interconnections between the nodes" Parrish (1996:2). Interactivity that encourages deeper cognitive processing (Craik \& Lockhart, 1972) will potentially lead to these interconnections being built.

\section{Interactivity as communication}

While the interactivity being considered in this paper relates specifically to that associated with the learner and computer, the term is also applied extensively to human-human communication, especially with respect to web based or online learning (Gilbert \& Moore, 1998). In identifying possible types of instruction as directive, content interactive, directed collaboration, collaborative and social they portray the various influences that impact the communication process in terms of teacher control, learner control and group influence. The extent to which distance plays a role in the success of the overall interactive process is also important. Moore (1989) proposed a transactional distance theory that suggested the more a teacher and student were separated the less effective the learning experience. This approached received significant discussion on the International Forum of Educational Technology \& Society (IFETS) online discussion list (IFETS, 1999), with considerable debate on the issue.

When considered in relation to computer enhanced learning, distance is an issue. While learner and computer are in close physical proximity, the extent to which there is distance (or lack of communication) between teacher, content and learner will diminish the effectiveness of the interactions. The extent to which communication or conversation between learner and designer can be integrated into a computer based medium has been analysed by Laurillard (1993), in which a conversational framework is used to identify the teacher-learner relationships. Enabling these concepts in the computer based medium is the challenge addressed within this paper. 


\section{Enabling effective interactivity}

Interactivity is perceived as both a function and outcome of the learning experience. It can be a manifestation of answering questions or an ingredient for successful learning. However, the complexity and sometimes fragmented descriptions of interactivity continue to provoke debate as to its essence. Because interactivity involves, at least theoretically, an interchange between learner and content, a relationship can be predicted between the effectiveness of computer based interactive experiences and a design strategy focusing on storytelling, play and narrative (Plowman, 1996a). This position is reinforced by Whitby (1993) who concludes:

Storytelling and narrative lie at the heart of all successful communication. Crude, explicit, button pushing interaction breaks the spell of engagement and makes it hard to present complex information that unfolds in careful sequence.

In this scenario the difference between the overt interactions are explicitly compared with the potential of narrative. The challenge confronting educational technology developers is the production of computer based environments that engage the learner in effective instructional communication without the interactivity interfering with the overall process. Identifying the concepts of storytelling and narrative as critical determinants of communication have been shown to provide a context to enable the potential of interactive learning environments.

\section{Interactivity and narrative}

\section{The impact of narrative}

The freedom associated with learner control can be restricted by the scripting of applications that require the user to adapt to their structure:

Since interactive interfaces ought to foster ... coordination between improvisation and planning we need to discover better theories of what is involved in the dynamic control of inquiry, line of thought, and action more generally. We need to discover more open ended models of coherence and narrative structure. Kirsh (1997:81)

Interactivity is portrayed as the crucial element of the new technology and yet recent research has demonstrated that there is still much to understand about the ways in which the interactive process facilities 
access to technology, especially in the context of computer based learning applications. Plowman (1996a:102-103) states,

\begin{abstract}
disruption of the narrative is strongest at the foci of interactivity ... (which) should be considered in terms of how they can be integrated into the overall narrative and how they can be used as a way of stimulating interest in the unfolding narrative ... by considering the interrelationship of narrative, linearity and interactivity and their design implications we can help learners to make sense of interactive multimedia.
\end{abstract}

The extent to which the interactions implemented are disruptive or constructive is an area that has received little attention to date but one that may provide some new guidelines for designers. As a concept, narrative can be viewed as a linear storyline or in terms of how the story is told, the way it is received, what meanings it can have and the specific social, cultural, gendered and technological context in which it is told (Plowman, 1996a; Humphreys, 1997b). But in what ways might narrative assist meaning, reduce the impact of interactive interference and provide the necessary framework to promote learning amongst diverse groups of learners? Plowman (1996a:92) suggests that:

\begin{abstract}
Narrative coherence is identified here with a lack of redundancy and a fixed sequence. Interactive multimedia (IMM) programmes challenge these traditional definitions of narrative because it can be suspended or altered at discrete decision points, the foci of interactivity, and a rearrangement of discrete elements gives rise to new text and new meanings. While the concepts of wholeness, unity and coherence of meanings are not fashionable in a post-modern world, in educational multimedia... the notion of multiple interpretations has different implications, particularly for comprehension and cognition.
\end{abstract}

While "narrative isn't just a shaping device: it helps us think, remember, communicate, and make sense of ourselves and the world" (Plowman, 1996a:3), when considered in terms of interactive environments, its perceived advantages are through enhancing comprehension and understanding:

Narrative structure is fundamental to comprehension to the extent that when it is clearly absent from certain forms of multimedia (it) can seriously undermine comprehension of the material. (Laurillard, 1998:231).

\title{
From narrative to play
}

Extending the concept of narrative and its impact on computer based learning, Humpherys (1997) explored the concept of play and play theory, suggesting that to maximise audience engagement through interactivity 
requires consideration of agency, narrative structure, emotional engagement and construction of meaning. In this context, play theory provides a:

Framework which accommodates the audience or user into the process of engagement with interactive media in ways that narrative theory finds difficult. ... Interactivity produces for the user of media a different relationship to story. This shift in relationship may be able to be framed as a shift from narrative, as an experience of recounting a story, to play, as an experience of enacting a story. Humpherys $(1997: 9,11)$

A focus on either narrative or play as structures to enhance engagement implies that the interactivity provided must be integrated to enable reinforcement of the specific learning objectives and to maintain the user's participation in the story. It is our challenge therefore to develop applications that minimise the potential for interactive interference. By considering the concept of interactivity, narrative and play in association with the links between the designer and learner, the following section presents a model to potentially enhance the communication between learner and designer within computer based environments.

\section{Interactivity on stage}

Linking the concept of narrative to interactivity is associated with other aspects of performance, such as theatre, illusion and magic (Laurel, 1990; Trognazzini, 1999). The following discussion identifies selections of work relevant to this area and the implications for developing more effective interactive encounters.

The association of education with theatre is by no means novel:

As we develop as persons, we develop a sophistication and sensitivity to
what is the "proper" role behaviour for various groups we must meet ...
We are in a sense able to predict the consequences of various behaviour
alternatives on others without actually performing them, and can select the
best role and performance ... This process is seen as "dramatic rehearsal"
$\ldots$ and is as complex as the relationship of actor and audience on stage.
Hodgkinson (1967:3)

In the context of education and social change, these concepts of performance, roles, identity and cues provide a focus for the learning environment. "As on stage, we are constantly sending out signals to those around us telling them how we wish to behave ... education at all levels is constantly faced with the problem of correcting misunderstood or 
unintentional cues" (Hodgkinson, 1967:22). To what extent therefore do computer based learning applications provide learners with a set of confusing cues, and in what way are learners allowed to play a role when working with educational technology? If, as Hodgkinson (1967) argues, being unaware of role playing can damage educational goals, it may be that the concept of roles, cues, theatre and performance hold clues as to making computer based learning work better.

Laurel (1990), who proposed the computer as theatre, also identifies the importance of human agency in human-computer interaction. Using this analogy, it is possible to extend the narrative and play concept to that of theatre and the role of the learner from observer or participant to actor:

\footnotetext{
By explicitly casting the language learner as actor (or other), a more playful and reflexive context for taking performative risks becomes possible. At the same time, the learner is pressed to assume responsibility for communicative acts that involve skill building at multiple levels of performance (phonological, kinetic, pragmatic), and that include but go beyond propositional knowledge. (Quinn, 1997:1).
}

If the learner is to become and actor, then the structure of the application can be perceived as a performance. Trognazzini (1999) identifies magicians and illusionists as performers and that, in the same way that their act must be believable, so must interactive software have the same elements of acceptance:

I propose that there is a "threshold of believability," a point at which careful design and meticulous attention to detail have been sufficient to arouse in the spectator or user a belief that the illusion is real. The exact point will vary by person and even by mood, so we must exceed it sufficiently to ensure believability.

If the learner believes the illusion created by the educational environment then the interactions will become integral to that illusion rather than as external controls to an environment being observed. Playing a role, being an actor in the learning process is not only about making choices but becoming part of the narrative, story or performance.

\section{The learner as actor}

Much of the western educational system is, or has been, based on the formal classroom environment and the dominant role of the teacher. I have little doubt that this system will prevail and acknowledge the extensive range of successful teacher-student interactions. However, the advent of computer based learning resources has extended the means by 
which learners can be emancipated from this environment. The discussion of interactivity, narrative and performance provides a context by which this emancipation may continue and develop. The following set of considerations are based on my preliminary findings from an as yet unpublished research study, and speculate that conceptualising the learner as actor may provide a means to enhance learner-designer communications.

To date, many computer based learning resources have placed the learner as observer (passive or active) in much the same way as members of an audience at a performance. However, this separation of actor and audience provides an essentially uni-directional communication - from stage to audience. If the learner were to take a position on stage, what might that mean for computer based learning and how might it be implemented?

Designers might extend the concepts proposed by Hannafin \& Peck (1988) to create scenarios in which the learner has an opportunity to participate. This would involve the learner being asked how familiar they are with the application and the extent to which they would like orientation to the application. The tour should allow the user to ask questions for clarification, in much the same way that an actor and director peruse the script and work through a series of rehearsals. Once comfortable with the location and prepared for "opening night", learners need to be made familiar with the controls (the stage, props and other actors) - not in their use but in their purpose - and the relevance of their appearance on the display (location on the stage). By using a narrative or story to define the performance in which the learner is participating, a logical and meaningful series of interactions can be employed.

An argument against such design structures is that they are too expensive or too difficult to implement, but this is not necessarily the case. If computer based learning resources continue to be used then it is important that they be effective. To date this has not been achieved consistently, so if more effective resource can be structured then the initial costs will be outweighed by the benefits. Similarly, as technology is developing so rapidly the creation of what now appear to be complex environments will become components of the development software.

The integration of the learner into the overall process may provide an environment in which the communication is focused on learner and teacher (designer) rather than learner and computer (content). Laurillard (1993) proposed the discursive, adaptive, interactive and reflective elements of education media in relation to effective teacher-learner 
communications. Continuing to develop the way in which people work with computers in learning contexts will provide the means by which these elements will continue to be successfully integrated into computer based environments. Conceptualising the learner as actor may provide a means to achieve this.

\section{Conclusion}

Interactivity in the context of computer based learning can be described in terms of different dimensions such as control, adaptation and communication. However, the range of interpretations of interactivity has done little to generate a common understanding or consistent implementation in computer based resources. Integrating current understandings of interactivity with the concept of narrative has demonstrated that further comprehension can be achieved in computer based environments. Extending this to the learner being an actor in a computer based or digital performance provides a strategy for developing a form of communication between learner and designer beyond that of the content structure embedded within an interface.

One of the first mottos I heard when beginning my career in this field was "you're only limited by your imagination". My vision is one of a learner integrated, engaged and achieving in a computer based environment imagining a learner on stage, playing a critical role in the narrative is how that vision might be realised. The success of computer based learning will be through interactivity as a manifestation of communication between designer and learner. If the designer can develop their ideas into a performance into which the learner is actor and interactivity the stage, then the illusion, magic and engagement so eagerly sought after might well be achieved.

\section{References}

Aldrich, F., Rogers, Y. \& Scaife, M. (1998). Getting to grips with "interactivity": Helping teachers assess the educational value of CD-ROMs. British Journal of Educational Technology, 29(4), 321-332.

Craik, F. \& Lockhart, R. (1972). Levels of processing: A framework for memory research. Journal of Verbal Learning \& Verbal Behavior, 11, 671-684.

Gilbert, L. \& Moore, D.R. (1998). Building interactivity into web courses: Tools for social and instructional interaction. Educational Technology, 38(3), 29-35.

Hannafin, M.J. (1989). Interaction strategies and emerging instructional technologies: Psychological perspectives. Canadian Journal of Educational Communication, 18(3), 167-179. 
Hannafin, M.J. \& Peck, K.L. (1988). The Design, Development, and Evaluation of Instructional Software. New York: Macmillan Publishing.

Hannafin, M.J., Hannafin, K.M., Hooper, S.R., Rieber , L.P. \& Kini, A.S. (1996). Research on and research with emerging technologies, in D.H. Jonassen (Ed.), Handbook of Research for Educational Communications and Technology. New York, NY: Simon \& Schuster.

Heath, J. (1995). When interactive media is not truly interactive. Active Learning, 3. http: / / www.cti.ac.uk/publ/actlea/issue3/heath/index.html [2 Dec 1999].

Hodgkinson, H.L. (1967). Education, Interaction and Social Change. Englewood Cliffs, NJ: Prentice-Hall.

Holmes, M. (1995). Interactivity primer: Exploring the essence of good interactivity. http:/ / www.multimediator.com/publications / write014.shtml [2 Dec 1999].

Humpherys, S. (1997). Audience engagement with interactive multimedia: An overview of four research projects. http: / / www.ngapartji.com.au/research/rosebud/research/audience_engage ment.html [28 July 1998].

International Forum of Educational Technology \& Society (1999). Transactional distance theory as a foundation for developing innovative and reactive instruction. http:/ / ifets.ieee.org/discussions/discuss.html [2 Dec 1999].

Iuppa, N.V. (1984). A Practical Guide to Interactive Video Design. White Plains, NY: Knowledge Industry Publications.

Jaspers, F. (1991). Interactivity or instruction? A reaction to Merrill. Educational Technology, 31(3), 21-24).

Kirsch, D. (1997). Interactivity and multimedia interfaces. Instructional Science, 25(2), 79-96.

Kristof, R. \& Satran, A. (1995). Interactivity by design: Creating and Communicating with New Media. Mountain View, CA: Adobe Press.

Laurel, B. (1991). Computers as Theatre. Reading, MA: Addison Wesley.

Laurillard, D. (1993). Rethinking University Teaching: A Framework for the Effective Use of Educational Technology. London: Routledge.

Laurillard, D. (1998). Multimedia and the learner's experience of narrative. Computers $\mathcal{E}$ Education, 31, 229-242.

Moore, M.G. (1989). Editorial: Three types of interaction. The American Journal of Distance Education, 3(2), 1-7.

Outing, S. (1998). What exactly is 'interactivity'? [2 Dec 1999]. http: / / www.mediainfo.com/ephome/news/newshtm/stop/st120498.htm 
Parrish, P. (1996). Interactivity in computer-aided learning (CAL).

http: / / www.comet.ucar.edu/pub_html/npi/interac3.html [2 Dec 1999].

Plowman, L. (1996a). Narrative, linearity and interactivity: Making sense of interactive multimedia. British Journal of Educational Technology, 27(2), 92-105.

Plowman, L. (1996b). Designing interactive media for schools: A review based on contextual observation. Information Design Journal, 8(3), 258-266.

Rhodes, D.M. \& Azbell, J.W. (1985). Designing interactive video instruction professionally. Training and Development Journal, December, 31-33.

Rose, E. (1999). Deconstructing interactivity in educational computing. Educational Technology, 39(1), 43-49.

Schaffer, L.C. \& Hannafin, M.J. (1986). The effects of progressive interactivity on learning from interactive video. Educational Communication and Technology Journal, 34(2), 89-96.

Schmeck, R.R. (1988). An introduction to strategies and styles of learning, in R.R. Schmeck (Ed), Learning Strategies and Learning Styles. New York: Plenum Press.

Schwier, R. \& Misanchuk, E. (1993). Interactive Multimedia Instruction. Englwood Cliffs, NJ: Educational Technology Publications.

Steinberg, E.R. (1991). Computer-Assisted Instruction: A Synthesis of Theory, Practice, and Technology. Hillsdale, NJ: Lawrence Erlbaum Associates.

Summers, J.A. (1990). Effect of interactivity upon student achievement, completion intervals, and affective perceptions. Journal of Educational Technology Systems, 19(1), 53-57.

Trognazzini, B. (1999). Magic and software design. http: / / www.asktog.com/ papers/magic.html [2 Dec 1999].

Weller, H.G. (1988). Interactivity in microcomputer-based instruction: Its essential components and how it can be enhanced. Educational Technology, 28(2), 23-27.

Whitby, M. (1993). Is interactive dead? http:/ / www.wired.com/wired/archive/1.01/interactive.html [2 Dec 1999].

\section{Rod Sims}

School of Multimedia and Information Technology

Southern Cross University

rsims@scu.edu.au 